\title{
Science Goals and Development of the Advanced Technology Solar Telescope
}

\author{
S. L. Keil, ${ }^{1}$ T. R. Rimmele,${ }^{1}$ J. Oschmann, ${ }^{2}$ R. Hubbard, ${ }^{1}$ \\ M. Warner, ${ }^{1}$ R. Price,${ }^{1}$ N. Dalrymple, ${ }^{1}$ and the ATST Team \\ ${ }^{1}$ National Solar Observatory, Sunspot, NM \& Tucson, Arizona, USA email: skeil@nso.edu \\ ${ }^{2}$ Ball Aerospace \& Technologies Corp., Boulder, CO.
}

\begin{abstract}
The Advanced Technology Solar Telescope (ATST) will perform high-resolution studies of the Sun's magnetic fields needed to understand their role in the fundamental processes responsible for solar variability. The generation of magnetic fields through dynamo processes, the amplification of fields through the interaction with plasma flows, and the destruction of fields remain poorly understood. There is incomplete insight as to what physical mechanisms are responsible for heating the corona, what causes variations in the radiative output of the Sun, and what mechanisms trigger flares and coronal mass ejections. Progress in answering these critical questions requires study of the interaction of the magnetic field and convection with a resolution sufficient to observe scales fundamental to these processes.

The 4-m aperture ATST is designed as a unique scientific tool, with excellent angular resolution, a large wavelength range, and low scattered light. With its integrated adaptive optics, the ATST will achieve a spatial resolution nearly 10 times better than any existing solar telescope.
\end{abstract}

\section{Introduction}

The Sun sustains life on Earth and impacts human endeavors in space through variations in its radiative, magnetic and particle output as caused by magnetic activity. The solar atmosphere is controlled by magnetic fields. The Advanced Technology Solar Telescope (ATST) is the first major instrument designed by the astronomical community in all of its aspects as a tool for magnetic remote sensing. Its collecting area, spatial resolution, wavelength performance and integral focal plane instrumentation are all targeted for understanding how magnetic fields affect the physical properties of the Sun Insights into the plama-field interactions on the Sun will bear directly on other problems in plasma and astrophysics.

The ATST will have the spatial, temporal, spectral resolution and dynamic range that is needed to see and measure the basic magnetic structures (magnetic fibrils) at the solar surface and into the outer atmosphere. Currently much of the magnetic field is invisible. We therefore need the ATST for quantifying, understanding, and predicting the consequences of such magnetism on solar-terrestrial and astrophysical plasmas. The need for the ATST was strongly advocated in the Astronomy and Astrophysics Decadal Survey (McKee \& Taylor, 2001) and the Solar and Space Physics Decadal Survey (Lanzerotti, 2003).

The ATST project is prepared to move into full-scale development, construction, and scientific exploitation of the ATST. The National Solar Observatory (NSO) is leading the ATST design effort, while other members of the ATST partnership are providing expertise in instrument development, polarimetry, modeling and theory, as well as advice and oversight to the project. The ATST program includes strong international participation 


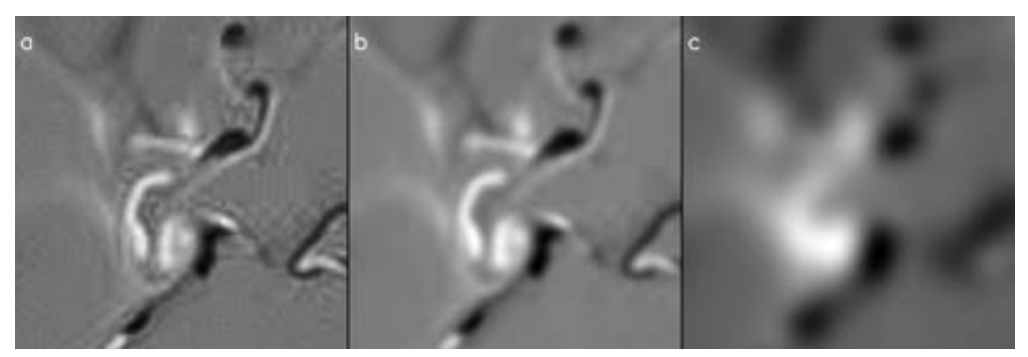

Figure 1. (a) A frame taken from a 3-D simulation (10-km grid) of solar magnetoconvection near the solar surface (courtesy of Fausto Cattaneo). Each box is $800 \mathrm{~km}$ on a side $(1.1 \mathrm{arcsec}$ ) and shows a snapshot of the magnetic field as it would appear looking down on the simulation. (b) The same fields seen through a diffraction limited 4-m telescope, and (c) through a diffraction-limited 1-m telescope (the best currently available). The model predicts rapidly evolving, highly mixed, bipolar fields that are twisted by strong turbulent downflows. The 4-m ATST is required to measure both the spatial and temporal characteristic of the the magnetic field associated with this small-scale dynamo process that is crucial to solar magnetism and to distinguish between competing models.

through its science and site survey working groups. In Europe, a consortium has formed to seek both national and European Union (EU) support for the ATST. Visit atst.nso.edu for a list of the groups participating in the ATST project and for lists of the science and site survey working group members.

The NSF-funded ATST Design and Development (D\&D) effort commenced in late 2001 and has produced a design with sufficient detail to allow a thorough assessment of ATST's feasibility and construction. The cost of construction and commissioning of the ATST is $\$ 161 \mathrm{M}$. This includes three major instruments, a fully integrated adaptive optics system, support facilities, approximately $20 \%$ contingency, and inflation adjustments to actual year of expenditure. Scientific operations are scheduled to begin in 2013.

\section{Science Drivers}

The ATST is designed to address the basic questions: What is the nature of solar magnetism; how does that magnetism control our star; and how can we model and predict its changing outputs that affect the Earth? Models of magnetoconvection predict that the Sun's magnetic fields coalesce and dissipate at spatial scales of a few tens of kilometers, well beyond the reach of current telescopes in space or on the ground. One such model is shown in figure 1 (Cattaneo, 1999). These elementary magnetic building blocks, or magnetic fibrils, are strongly linked to the Sun's properties as a variable magnetic star. The unambiguous measurement of magnetic fibril characteristics and the buffeting of these fibrils by turbulence, the coalescence of fields into strong concentrations such as pores and sunspots, their role in transporting and dissipating energy, the resulting solar activity, and the validation or rejection of models for these processes are major goals of the ATST.

The photosphere is a crucial region where energy is readily transformed from convective motion into thermal and magnetic energy, and electromagnetic radiation. The sunspot region shown in figure 2 is a good example of this complex interaction. Rimmele (2004) illustrates the state-of-the-art in observing plasma flow and magnetic fields at the diffraction limit using adaptive optics and demonstrates the need for even higher resolution and more light gathering power to fully measure the magnetic field. The energy 


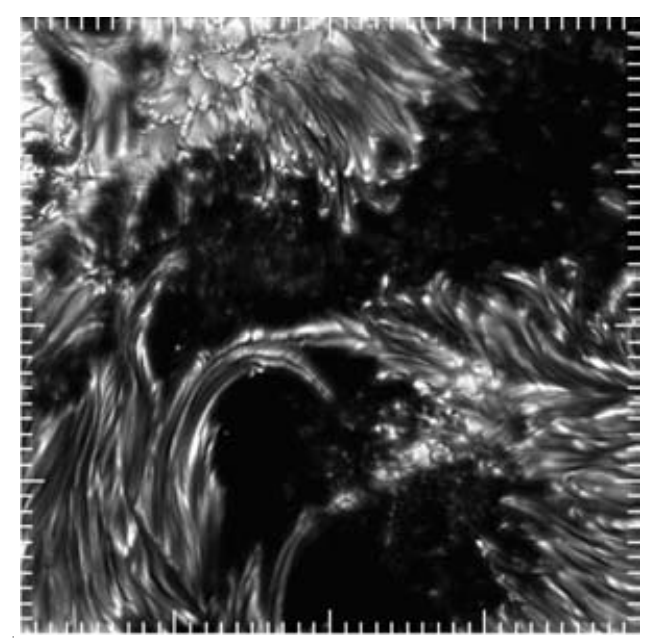

Figure 2. Part of an active region observed in the $\mathrm{CH}$ bandhead at $430.5 \mathrm{~nm}$ (G-band) at the diffraction limit of the $76 \mathrm{~cm}$ Dunn Solar Telescope at NSO Sacramento Peak using the high-order adaptive optics system (courtesy of Thomas Rimmele). Tick marks are at 1 arcsec intervals. The filamentary structure, dark channels, and bright points are all manifestations of the magnetic field and are mostly unresolved. Current meter class telescopes cannot provide the resolution and they do not collect sufficient photons to measure the fields associated with these features before they evolve. Only with the ATST will we be able to collect precision polarimetric data with a temporal cadence necessary to capture the evolution of these fine structures and finally understand their physical origin.

stored in magnetic fields is eventually dissipated at higher layers of the solar atmosphere, sometimes in the form of violent flares and coronal mass ejections (CMEs) that ultimately affect the Earth and drive space weather. The photosphere, the chromosphere, transition region, and the corona are connected through the magnetic field and therefore have to be treated as one system, rather than as individual layers. The ATST is a crucial tool to understand this complex, interconnected physical system.

ATST observations will answer many long-standing questions that are essential to the physics of solar activity and variability:

- What role does the ubiquitous small-scale solar magnetic flux play in the generation of solar magnetism leading to cyclic variation of magnetic activity?

- How do strong field concentrations (pores and sunspots) form, and how do they modify the turbulent convection?

- How does the magnetic field carry energy into the solar atmosphere, making the Sun a variable X-ray star?

- How do magnetic fields drive solar irradiance changes (especially those that impact the terrestrial climate)?

- How is magnetic energy stored and then released catastrophically?

- What is the role of magnetic helicity (twist) and chirality (handedness) in the solar dynamo and in solar activity?

To answer these questions, we must investigate the energy transport and conversion processes in magnetized plasma to establish:

- How mass motions and magnetic field interact in the Sun.

- How the magnetic field emerges through the solar surface and how it disappears. 
Table 1. Science Goals Leading to Observation requirements and Technical Specifications.

\begin{tabular}{|c|c|c|c|c|}
\hline Science Goals & $\begin{array}{l}\text { Observational } \\
\text { Requirements }\end{array}$ & $\begin{array}{l}\text { Telescope } \\
\text { Requirements }\end{array}$ & $\begin{array}{l}\text { Telescope and Site } \\
\text { Requirements }\end{array}$ & Instruments \\
\hline $\begin{array}{l}\text { - Small-scale dynamo } \\
\text { - Origin of weak field and } \\
\text { importance for solar } \\
\text { cycle } \\
\text { - Magnetoconvection } \\
\text { - Flux emergence and } \\
\text { dissipation } \\
\text { - Formation, destruction, } \\
\text { internal structure of flux } \\
\text { tubes and sheets } \\
\text { - Structure of sunspots } \\
\text { - Generation of MHD and } \\
\text { acoustic oscillations } \\
\text { - Chromospheric and } \\
\text { coronal heating } \\
\text { - Triggering of activity }\end{array}$ & $\begin{array}{l}\text { - Observe fundamental } \\
\text { astrophysical } \\
\text { processes over scales } \\
\text { at which they occur } \\
\text { - Measure B and } \\
\text { plasma parameters } \\
\text { with sufficient spatial } \\
\text { and temporal resolu- } \\
\text { tion and sufficient } \\
\text { accuracy to test } \\
\text { theoretical models } \\
\text { - Observe visible and } \\
\text { near-infrared } \\
\text { spectrum }\end{array}$ & $\begin{array}{l}\text { - Spatial resolution } \\
\text { - 0.03" at } 500 \mathrm{~nm} \\
\text { - 0.1" at } 1600 \mathrm{~nm} \\
\text { - Large photon collecting } \\
\text { area } \\
\text { - Accurate polarimetry } \\
\text { (better than } 10^{-4} \text { ) } \\
\text { - Low scattered light }\end{array}$ & $\begin{array}{l}\text { - Aperture: } 4-\mathrm{m} \\
\text { - Seeing control: } \\
\text { - Adaptive optics } \\
\text { - Thermal control } \\
\text { - Low and stable } \\
\text { instrumental } \\
\text { polarization }<1 \% \\
\text { - Excellent seeing sight }\end{array}$ & $\begin{array}{l}\text { - Visible/NIR } \\
\text { spectrographs and } \\
\text { polarimeters } \\
\text { - Visible/NIR narrow- } \\
\text { band filters } \\
\text { - Visible/NIR } \\
\text { detectors } \\
\text { - UV Polarimetry }\end{array}$ \\
\hline $\begin{array}{l}\text { - Structure and dynamics } \\
\text { of upper atmosphere } \\
\text { - Shock wave heating } \\
\text { - COmsphere } \\
\text { - Spicules } \\
\text { - MHD wave and } \\
\text { - topological heating } \\
\text { - Prominence formation } \\
\text { and eruption } \\
\text { - Flares } \\
\text { - CMEs } \\
\text { - 3D structure of } \\
\text { magnetic field: } \\
\text { - Chromosphere and } \\
\text { coronal magnetic } \\
\text { fields }\end{array}$ & $\begin{array}{l}\text { - Measure B and } \\
\text { plasma parameters in } \\
\text { upper atmospheric } \\
\text { layers } \\
\text { - Observe: } \\
\text { - CO lines at } 4800 \mathrm{~nm} \\
\text { (transition zone from } \\
\beta>1 \text { to } \beta<1, \mathrm{~T} \\
\text { diagnostics } \\
\text { - Mg I at } 12000 \mathrm{~nm} \\
\text { (magnetic field, } \\
\text { upper photosphere) } \\
\text { - He I } 1083 \mathrm{~nm} \\
\text { (Magnetic field, } \\
\text { chromosphere) } \\
\text { - Coronal lines at } \\
\text { 1074.7 nm and } 3900 \\
\text { nm, magnetic fields, } \\
\text { corona) }\end{array}$ & $\begin{array}{l}\text { - High resolution } \\
\text { - IR access }(>12000 \mathrm{~nm}) \\
\text { - Large photon flux } \\
\text { - Accurate polarimetry } \\
\text { - Low scattered light } \\
<10^{-5} \text { at } 1.1 \text { solar } \\
\text { radii Coronagraphic } \\
\text { capabilities in IR }\end{array}$ & $\begin{array}{l}\text { - Aperture: } 4-\mathrm{m} \text { or } \\
\text { larger } \\
\text { - Open-air design } \\
\text { - Dust control } \\
\text { - Adaptive optics } \\
\text { - Unobscured light } \\
\text { path } \\
\text { - Low sky brightness }\end{array}$ & $\begin{array}{l}- \text { NIR/thermal IR } \\
\text { detectors } \\
\text { - NIR/Thermal IR } \\
\text { spectrograph and } \\
\text { polarimeter }\end{array}$ \\
\hline $\begin{array}{l}\text { - Active region evolution } \\
\text { - Understand process of } \\
\text { activity build-up and } \\
\text { triggering } \\
\text { - Dynamo processes }\end{array}$ & $\begin{array}{l}\text { - Measure B and } \\
\text { plasma parameters at } \\
\text { different layers in } \\
\text { atmosphere and over } \\
\text { entire active region } \\
\text { - Long time series }\end{array}$ & $\begin{array}{l}\text { - Large FOV } \\
\text { - High resolution over } \\
\text { large FOV }\end{array}$ & $\begin{array}{l}\text { - AO \& site with large } \\
\text { isoplanatic patch or } \\
\text { - MCAO }\end{array}$ & - All of the above \\
\hline $\begin{array}{l}\text { - Explore the unknown } \\
\text { - New discoveries }\end{array}$ & $\begin{array}{l}\text { - e.g., Explore IR } \\
\text { spectrum }>12000 \mathrm{~nm} \\
\text { - Flare spectra in IR }\end{array}$ & - Flexibility, adaptability & $\begin{array}{l}\text { - Multi-observing } \\
\text { stations } \\
\text { - Ability to implement } \\
\text { new ideas }\end{array}$ & $\begin{array}{l}\text { - All of the above } \\
\text { - User furnished }\end{array}$ \\
\hline
\end{tabular}

- How magnetic reconnection/annihilation works.

- How micro-scale instabilities lead to large-scale effects.

Achieving the ATST scientific goals will answer the fundamental questions raised above. The observational requirements that will achieve these science goals were developed in the ATST Science Requirements Document (SRD) (http://atst.nso.edu) by the ATST Science Working Group with substantial inputs from the solar community. Table 1 summarizes these key goals and their translation into requirements.

Magnetic fields control the static and dynamic structure of the solar corona, and ATST, for the first time, will allow us to measure this "dark energy," which has been largely invisible with all current ground and space experiments. With careful attention to scattered light performance and by optimizing the facility for infrared capabilities, we will finally be able to see the coronal magnetic fields, which are responsible for so much of the Sun's variability.

Understanding solar magnetism and developing models of its behavior drives many observational requirements. We need to observe the weak field in the photosphere, which requires high polarimetric sensitivity and high spatial resolution $(<0.1$ arcsec $)$. We must observe the vector magnetic fields at high spatial and temporal resolution to determine their twist and its source, the types of waves being generated and dissipated, and how reconnection takes place. We need to understand the structure of the small-scale field and 

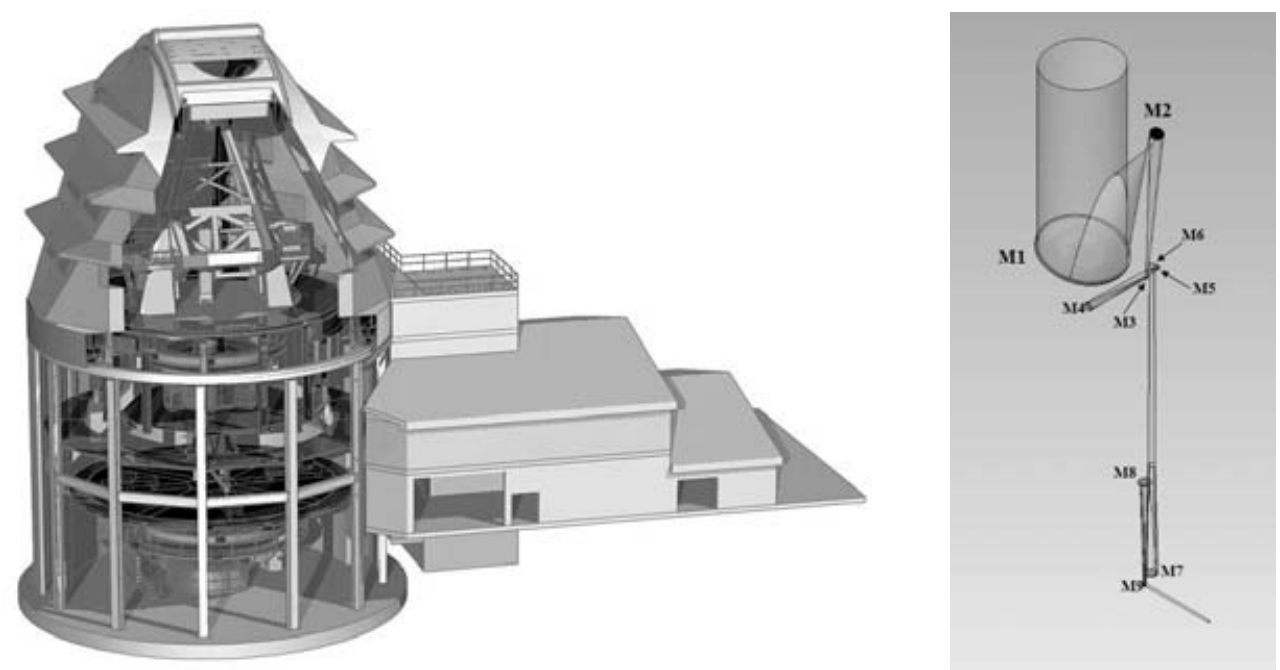

Figure 3. (left) A cut-away showing the current overall concept for the ATST. (right) Optical layout up to the feed for the coudé platform.

how much flux is hidden within the resolution element of existing telescopes. The position and geometry of the field in the outer atmosphere is inferred from brightness images showing loops, or from extrapolations of photospheric field. We must observe the chromospheric and coronal vector magnetic fields associated with these loops. These observations drive spatial resolution, polarimetric sensitivity, access to the IR, coronagraphic capabilities, and temporal cadence. The ATST design described below will provide the required observational capability.

\section{Technical Approach}

The Advanced Technology Solar Telescope (ATST) is an all-reflecting, four-meter, offaxis Gregorian telescope housed in a co-rotating, ventilated and cooled dome. The technical challenge of designing a large aperture telescope for solar observations are discussed in Rimmele et al. (2002). The ATST delivers a 300-arcsec field of view (unvignetted over a 240 arcsec square) to a Gregorian and coudé observing stations. Energy outside of this field is rejected from the system by a heat stop located at prime focus, allowing manageable thermal loading on the optical elements that follow. The telescope also includes an integrated adaptive optics system (Rimmele et al., 2004) designed to provide diffraction-limited images to the focal-plane instruments at the coudé observing stations. It is an open system, providing broad spectral coverage from the near ultraviolet to the thermal infrared. ATST is desinged to have the lowest scattered light performance of any large-aperture telescope ever constructed. Its coronagraphic properties, especially at infrared wavelengths, allow it to observe the solar corona far from the solar disk. Figure 3 shows the overall concept and optical layout for the ATST.

The ATST optical design has two features that distinguish it from nighttime telescopes with similar aperture size: it is off axis and Gregorian. Both of these features are included in the design in direct response to the science requirements. Figure 3 summarizes the optical design.

Four mirrors are required to transfer the beam over the mechanical altitude and azimuth axes. These mirrors are designated M3 through M6. M3 and M6 are flat folding 


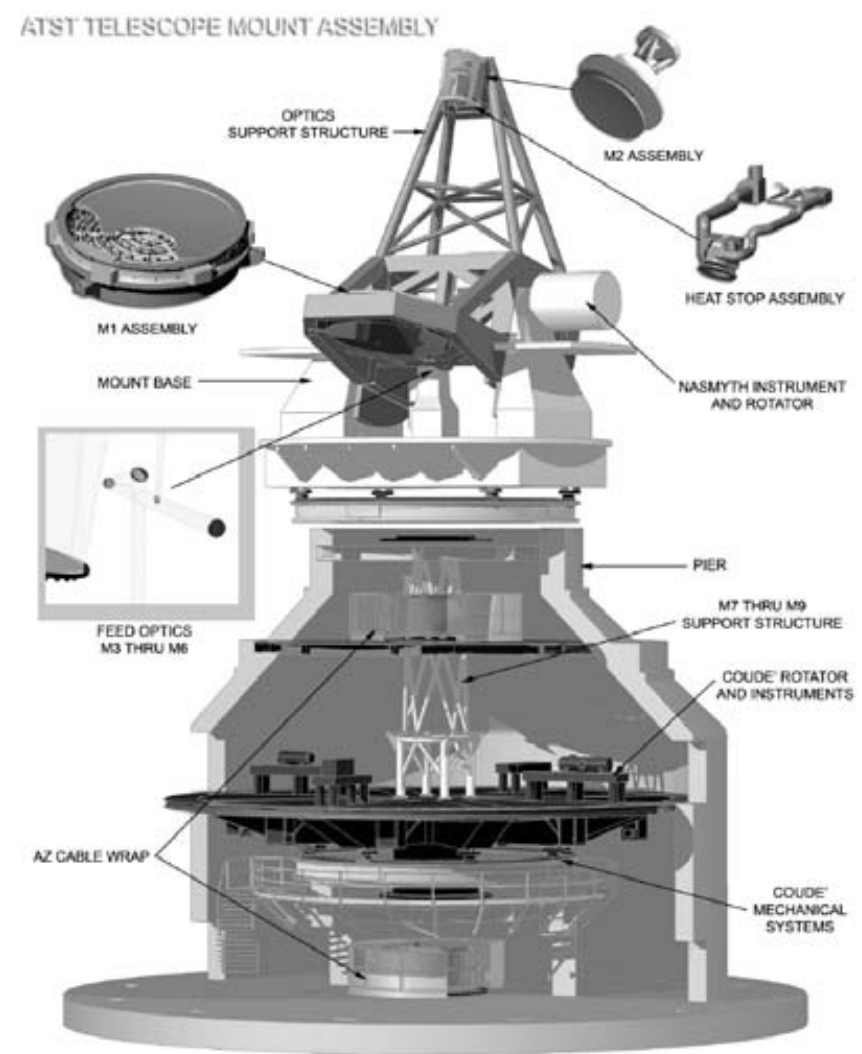

Figure 4. Telescope Assembly

mirrors. M4 is an off-axis conic that serves to re-image the Gregorian focus over the mechanical axis of the telescope and down into the coudé lab area. M5 is a nominally flat fold mirror that also serves as the deformable mirror for the high-order adaptive optics system. M6 also provides fast tip-tilt correction. M7 collimates the beam, M8 is a nearly flat corrector, and M9 is a pupil-steering mirror that can feed multiple observing stations.

The decision to enclose the telescope resulted from early trade studies of enclosed vs. open or retractable dome concepts. Main issues included achieving good flushing of the local seeing around the telescope, which is achieved by the ambient wind in ideal conditions on telescopes such as the Dutch Open Telescope. However, the DOT is a 0.5$\mathrm{m}$ mirror and is supported by a very stiff mount. For the 4-m ATST primary mirror, wind loading can affect the performance and achieving sufficient stiffness to avoid this is very costly and can even introduce additional seeing due to heating of the telescope structure. The concept developed to address these issues is a highly ventilated, co-rotating hybrid enclosure with active thermal control. The concept provides good wind throughput and rapid flushing of the air in the enclosure.

Some of the enclosure functions include:

- Passive ventilation for local seeing control

- Wind throttling under high-wind conditions 
- Active ventilation under low-wind conditions

- Dust infiltration control (during coronal observations)

- Shading of structures and weather protection

The shades over the vents (figure 3) prevent direct sunlight from entering the enclosure and heating the interior or telescope structure. They also help direct wind that might skip over the dome to flow through it. When fully open, the vents attenuate the ambient wind speed by approximately 40-70\%, depending on the angle of incidence. Under high wind conditions, the vents can be closed down to allow a controlled flow across the telescope. During coronal observations when dust is a more important factor than spatial resolution, the vents can be closed.

The full telescope assembly is shown in figure 4. It is comprised of (1) the Telescope Mount Assembly; (2) the M1 Assembly; (3) the Heat Stop Assembly; (4) the M2 Assembly; (5) the Feed Optics; (6) the Baffles and Stops; and (7) the System Interconnects.

Wherever possible, the design of the telescope assembly and its subcomponents were based on previous successful large telescope systems, including the structural layout, the servo and control systems, the pier concept, and many of the mechanical subsystems. Where it was impossible to emulate existing telescope designs, we have verified the ATST design by various methods including full static and dynamic finite element (FE) studies of the overall structure and pier. Transient thermal analyses, computational fluid dynamics (CFD) studies, and various other calculations have been performed as well to support the design.

The Telescope Mount Assembly (TMA) provides structural support for the major optics and instruments of the ATST observatory. It includes a variety of mechanical subassemblies, bearings, controllers, drives, and equipment that are used to point, track, and slew these optics and instruments during science observations. The TMA is comprised of five major components: (1) the mount and drive system; (2) the coudé rotator and drive system; (3) the pier; (4) the ancillary mechanical systems; and (5) the mount control system.

The coudé rotator is the large steel assembly inside the pier that rotates about an azimuth axis coincident with the telescope mount. It provides mounting interfaces for the beam-reducing relay optics and all of the coudé-level instrumentation of ATST. The rotator can accommodate the simultaneous installation of up to eight instruments of $3000 \mathrm{~kg}$ each. The flooring system is designed to allow personnel to work around the instruments during normal observations.

As a telescope system, ATST's focal plane instrumentation is fundamental to its scientific performance and mission. ATST requires its spectropolarimetric imaging instrumentation to achieve exceptional magnetic remote sensing capabilities. Thus, visible and near-infrared spectropolarimeters, as well as visible tunable filters, are essential instruments and must be developed during the construction phase. Additional instruments, such as an IR tunable filter, IR fiber spectrographs, and UV spectropolarimeters, will be developed as part of the ongoing ATST instrument program after commissioning and during operation

\section{Summary}

The ATST project is structured in two phases, the on-going Design and Development (D\&D), and Construction and Commissioning. The D\&D phase spans FY 2001-2005 and is funded at $\$ 10.9$ million. It produced the baseline design and in FY2004 will lead to final selection of the best possible site for the ATST based on seeing, solar visibility, and costs. The D\&D phase has engaged a large portion of the solar physics community. Along with 
the National Solar Observatory, major support and cost sharing during the D\&D phase has come from the High Altitude Observatory, the New Jersey Institute of Technology, the University of Hawaii, and the Air Force Research Laboratory. The more than twenty collaborating institutions are providing additional support in developing science goals and requirements, guiding the site survey, and instrument design development. Other potential national and international cost-sharing partners have participated in the various working groups, instrument design, and reviews. Major contributors include scientists from the Instituto de Astrofisica de Canarias in Spain, the Kiepenheuer-Institut für Sonnenphysik in Germany, ETH Zürich Institue of Astronomy in Switzerland, and the Università di Napoli and the Osservatorio Astrofisico di Arcetri in Italy.

The Construction phase will span FY 2006-2013. Science operations will begin in 2012, ramping up to full operations in 2013 . The total cost of $\$ 161$ million (including inflation and contingency) covers the 4-meter ATST and its first set of science instruments. Before the 2006 commencement of the construction phase, national and international partnerships to cost-share the ATST with NSF will be developed.

The ATST offers tremendous opportunity for the training of students and recruitment of post-docs and faculty in solar physics who will become users of the ATST and the instrument builders and theoreticians of the future. The ATST program has and will continue to actively involve large segments of the US and international solar physics community, helping to strengthen solar astronomy programs at universities and national centers.

\section{Acknowledgements}

The National Science Foundation (NSF) through the National Solar Observatory (NSO) funds the ATST Project. The NSO is operated under a cooperative agreement between the Association of Universities for Research in Astronomy, Inc. (AURA) and NSF.

The ATST represents a collaboration of 22 institutions, reflecting a broad segment of the solar physics community. The NSO is the Principal Investigator (PI) institution, and the co-PI institutions are the High Altitude Observatory, New Jersey Institute of Technology's Center for Solar Research, University of Hawaii's Institute for Astronomy, and the University of Chicago Department of Astronomy and Astrophysics.

\section{References}

Cattaneo, F. 1999 Ap. J. 515, pp L39-L42

Lanzerotti, L. 2003 Solar and Space Physics Survey Committee, National Research Council (2003). The Sun to the Earth - and Beyond: A Decdal Research Strategy in Solar and Space Physics, Washington, DC: National Academy Press.

Mckee, C. \& Taylor, J. 2001 Astronomy and Astrophysics Survey Committee, Board on Physics and Astronomy, Space Studies Board, National Research Council (2001). Astronomy and Astrophysics in the New Millennium, Washington, DC: National Academy Press.

Rimmele, T., Keil, S., Keller, C., Hill, F., Briggs, J., Dalrymple, N., Goodrich, B., Hegwer, S., Hubbard, R., Oschmann, J., Radick, R., Ren, D., Wagner, S., Wampler, S., Warner, M, and the ATST Team. 2002 In Large Ground-based Telescopes (ed. J. Oschmann \& L. Stepp), Proceedings SPIE 4837, pp 94-108

Rimmele, T. 2004 Ap. J. 604, pp 906-923

Rimmele, T., Richards, K., Hegwer, S., Gregory, S., Moretto, G., Didkovsky, L., Goode, P., Langlois, M., Marion, J., \& Marquette, W. 2004 Proceedings SPIE 5490, in press 